\title{
The effectiveness of a low-fat vegan diet for the prevention and management of type 2 diabetes: A systematic review
}

\author{
Alex Webb and Katie Lane \\ Liverpool John Moores University, Liverpool, United Kingdom
}

\section{Abstract}

Global diabetes prevalence is projected to rise from 382 million in 2013 to 592 million in 2030 with type 2 diabetes (T2D) accounting for $90-95 \%$ of cases ${ }^{(1,2)}$. Approximately 4.7 million people in the UK had diabetes in 2018 , this is estimated to rise to over 5 million by $2025^{(2)}$ costing the NHS $£ 15.1$ bn by $2035^{(3)}$.

Low-fat vegan (LFV) diets are associated with improvements in T2D biomarkers ${ }^{(4)}$ however, to date no systematic review has evaluated the effectiveness of LFV diets in prevention and management of T2D. The aim of this study was to assess effects of LFV diets on T2D biomarkers in controlled trials for those with, or at risk of developing T2D.

The review followed the PRISMA checklist for systematic reviews and used Cochrane Collaboration search strategies, which support the identification of randomised controlled trials (RCT's) ${ }^{(5)}$. Searches were conducted and titles and abstracts screened for relevance by two independent review authors (AW or KEL). Studies that evaluated a LFV diet to reduce the risk of, or manage T2D using controlled trials were included with publication range from January 2003 to June 2018.

Search criteria identified 130 articles, which reduced to 21 upon abstract and title screening. Further application of inclusion/exclusion criteria led to identification of six key studies. Four studies were RCT's, and two were cluster-controlled trials at corporate sites, one of which was randomised. In three of the studies participants were previously diagnosed with T2D, in two of the studies participants had a BMI $\geq 25 \mathrm{~kg} / \mathrm{m}^{2}$ and/or were previously diagnosed with T2D, and one study had participants with BMI between 28 and $40 \mathrm{~kg} / \mathrm{m}^{2}$. Four studies had a control group (non-intervention) two of which had a conventional diabetes diet (American Dietetic Association $^{(6)}$ ) as a comparison group. Mean study length was 28.3 weeks; ranging from 16-74 weeks. An intention-to-treat and medication adjusted analysis was carried out; significant effects for BMI, body weight, HbAlc, fasting plasma glucose, LDL, HDL and total cholesterol were reported in most of the studies.

Results from this systematic review demonstrate that a LFV diet is an effective alternative therapy to improve T2D biomarkers in those with, or at elevated risk of developing the disease. The LFV diet showed consistent positive effects on T2D biomarkers. Its effects are comparable to conventional T2D diets and energy restrictive diets, despite absence of restrictions on energy intake or portion sizes.

\section{Conflict of Interest}

There is no conflict of interest 